\title{
Formally Verified Montgomery Multiplication
}

\author{
Christoph Walther ${ }^{(凶)}(\mathbb{D}$ \\ Technische Universität Darmstadt, \\ Darmstadt, Germany \\ Chr.Walther@informatik.tu-darmstadt.de
}

\begin{abstract}
We report on a machine assisted verification of an efficient implementation of Montgomery Multiplication which is a widely used method in cryptography for efficient computation of modular exponentiation. We shortly describe the method, give a brief survey of the Verifun system used for verification, present the formal proofs and report on the effort for creating them. Our work uncovered a serious fault in a published algorithm for computing multiplicative inverses based on NewtonRaphson iteration, thus providing further evidence for the benefit of computer-aided verification.
\end{abstract}

Keywords: Modular arithmetic $\cdot$ Multiplicative inverses Montgomery Multiplication · Program verification Theorem proving by induction

\section{Introduction}

Montgomery Multiplication [6] is a method for efficient computation of residues $a^{j} \bmod n$ which are widely used in cryptography, e.g. for RSA, Diffie-Hellman, ElGamal, DSA, ECC etc. [4,5]. The computation of these residues can be seen as an iterative calculation in the commutative ring with identity $R_{n}=$ $\left(\mathbb{N}_{n}, \oplus, \mathfrak{i}_{n}, \odot, 0,1 \bmod n\right)$ where $n \geq 1, \mathbb{N}_{n}=\{0, \ldots, n-1\}$, addition defined by $a \oplus b=a+b \bmod n$, inverse operator defined by $\mathfrak{i}_{n}(a)=a \cdot(n-1) \bmod n$, multiplication defined by $a \odot b=a \cdot b \bmod n$, neutral element 0 and identity $1 \bmod n$.

For any $m \in \mathbb{N}$ relatively prime to $n$, some $m_{n}^{-1} \in \mathbb{N}_{n}$ exists such that $m \odot m_{n}^{-1}=1 \bmod n . m_{n}^{-1}$ is called the multiplicative inverse of $m$ in $R_{n}$ and is used to define a further commutative ring with identity $R_{n}^{m}=\left(\mathbb{N}_{n}, \oplus, \mathfrak{i}_{n}, \otimes, 0\right.$, $m \bmod n$ ) where multiplication is defined by $a \otimes b=a \odot b \odot m_{n}^{-1}$ and identity given as $m \bmod n$. The multiplication $\otimes$ of $R_{n}^{m}$ is called Montgomery Multiplication.

The rings $R_{n}$ and $R_{n}^{m}$ are isomorphic by the isomorphism $h: R_{n} \rightarrow R_{n}^{m}$ defined by $h(a)=a \odot m$ and $h^{-1}: R_{n}^{m} \rightarrow R_{n}$ given by $h^{-1}(a)=a \odot m_{n}^{-1}$. Consequently $a \cdot b \bmod n$ can be calculated in $\operatorname{ring} R_{n}^{m}$ as well because

$$
a \cdot b \bmod n=a \odot b=h^{-1}(h(a \odot b))=h^{-1}(h(a) \otimes h(b)) .
$$

(C) The Author(s) 2018

H. Chockler and G. Weissenbacher (Eds.): CAV 2018, LNCS 10982, pp. 505-522, 2018.

https://doi.org/10.1007/978-3-319-96142-2_30 


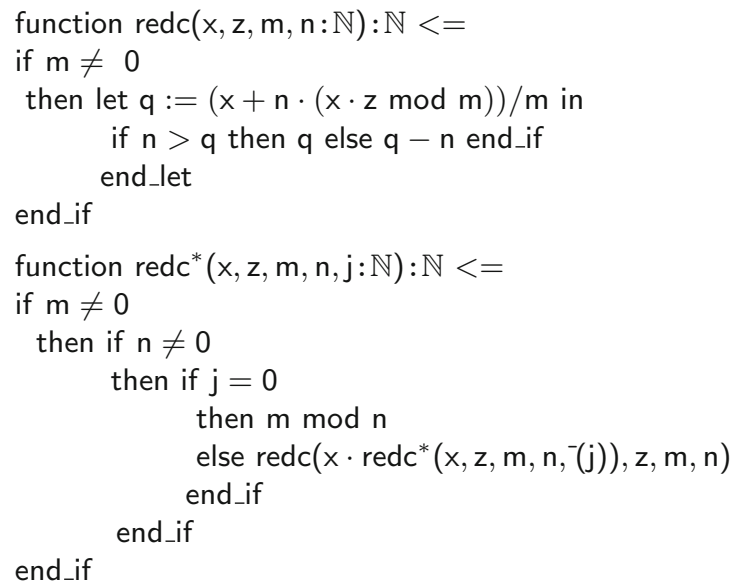

Fig. 1. Procedures redc and redc* implementing the Montgomery Reduction

The required operations $h, \otimes$ and $h^{-1}$ can be implemented by the so-called Montgomery Reduction redc [6] (displayed in Fig. 1) as stated by Theorem 1:

Theorem 1. Let $a, b, n, m \in \mathbb{N}$ with $m>n>a, n>b$ and $n, m$ relatively prime, let $I=\mathfrak{i}_{m}\left(n_{m}^{-1}\right)$ and let $M=m^{2} \bmod n$. Then $I$ is called the Montgomery Inverse and (1) $h(a)=\operatorname{redc}(a \cdot M, I, m, n)$, (2) $a \otimes b=\operatorname{redc}(a \cdot b, I, m, n)$, and (3) $h^{-1}(a)=\operatorname{redc}(a, I, m, n)$.

By $(*)$ and Theorem $1, a \cdot b \bmod n$ can be computed by procedure redc and consequently $a^{j} \bmod n$ can be computed by iterated calls of redc (implemented by procedure redc* of Fig. 1) as stated by Theorem 2 :

Theorem 2. Let a, n, $m, I$ and $M$ like in Theorem 1. Then for all $j \in \mathbb{N}:^{1}$

$$
a^{j} \bmod n=\operatorname{redc}\left(\operatorname{redc} c^{*}(\operatorname{redc}(a \cdot M, I, m, n), I, m, n, j), I, m, n\right) .
$$

By Theorem $2, j+2$ calls of redc are required for computing $a^{j} \bmod n$, viz. one call to map a to $h(a), j$ calls for the Montgomery Multiplications and one call for mapping the result back with $h^{-1}$. This approach allows for an efficient computation of $a^{j} \bmod n$ in $R_{n}^{m}$ (for sufficient large $j$ ), if $m$ is chosen as a power of 2 and some odd number for $n$, because $x \bmod m$ then can be computed with constant time and $x / m$ only needs an effort proportional to $\log m$ in procedure redc, thus saving the expensive $\bmod n$ operations in $R_{n}$.

\footnotetext{
${ }^{1}$ Exponentiation is defined here with $0^{0}=1$ so that $\operatorname{redc}\left(\operatorname{redc} c^{*}(\operatorname{redc}(0 \cdot M, I, m, n), I\right.$, $m, n, 0), I, m, n)=1 \bmod n$ holds in particular.
} 


\section{About Verifun}

The truth of Theorems 1 and 2 is not obvious at all, and some number theory with modular arithmetic is needed for proving them. Formal proofs are worthwhile because correctness of cryptographic methods is based on these theorems.

$\begin{array}{lll}\text { structure bool } & <= & \text { true, false } \\ \text { structure } \mathbb{N} & <= & { }^{+}\left({ }^{-}: \mathbb{N}\right) \\ \text { structure signs } & <= & +^{\prime},{ }^{\prime} \\ \text { structure } \mathbb{Z} & <= & {[\text { outfix] }\langle:\rangle(\text { sign:signs, }[\text { outfix] } \mid: \mathbb{N})} \\ \text { structure triple[@T1, @T2, @T3] } & <=\left[\text { outfix] } \lessdot:>\left([\text { postfix }]_{1}: @ T 1,\right.\right. \\ & & \left.[\text { postfix }]_{2}: @ T 2,[\text { postfix }]_{3}: @ T 3\right)\end{array}$

lemma $\mathrm{z} \neq 0 \rightarrow[\mathrm{x} \cdot(\mathrm{y} \bmod \mathrm{z}) \equiv \mathrm{x} \cdot \mathrm{y}] \bmod \mathrm{z}<=\forall \mathrm{x}, \mathrm{y}, \mathrm{z}: \mathbb{N}$ if $\{\neg \mathrm{z}=0,(\mathrm{x} \cdot(\mathrm{y} \bmod \mathrm{z}) \bmod \mathrm{z})=(\mathrm{x} \cdot \mathrm{y} \bmod \mathrm{z})$, true $\}$

Fig. 2. Data structures and lemmas in $\sqrt{\text { eriFun }}$

Proof assistants like Isabelle/HOL, HOL Light, Coq, ACL2 and others have been shown successful for developing formal proofs in Number Theory (see e.g. [14]). Here we use the VeriFunsystem ${ }^{2}[7,10]$ to verify correctness of Montgomery Multiplication by proving Theorems 1 and 2. The system's object language consists of universal first-order formulas plus parametric polymorphism. Type variables may be instantiated with polymorphic types. Higher-order functions are not supported. The language provides principles for defining data structures, procedures operating on them, and for statements (called "lemmas") about the data structures and procedures. Unicode symbols may be used and function symbols can be written in out-, in-, pre- and postfix notation so that readability is increased by use of the familiar mathematical notation. Figure 2 displays some examples. The data structure bool and the data structure $\mathbb{N}$ for natural numbers built with the constructors 0 and ${ }^{+}(\ldots)$ for the successor function are the only predefined data structures in the system. ${ }^{-}(\ldots)$ is the selector of ${ }^{+}(\ldots)$ thus representing the predecessor function. Subsequently we need integers $\mathbb{Z}$ as well which we define in Fig. 2 as signed natural numbers. For instance, the expression $\left\langle^{\prime}-', 42\right\rangle$ is a data object of type $\mathbb{Z}$, selector sign yields the sign of an integer (like '-' in the example), and selector $\mid .$. | gives the absolute value of an integer (like 42 in the example). Identifiers preceded by @ denote type variables, and therefore polymorphic triples are defined in Fig. 2 . The expression $\lessdot 42,\left\langle{ }^{\prime}+', 47\right\rangle,\left\langle{ }^{6}-,, 5\right\rangle>$ is an example of a data object of type triple $[\mathbb{N}, \mathbb{Z}, \mathbb{Z}]$. The $i^{\text {th }}$ component of a triple is obtained by selector $(\ldots)_{i}$.

Procedures are defined by if - and case-conditionals, functional composition and recursion like displayed in Fig. 1. Procedure calls are evaluated eagerly,

\footnotetext{
${ }^{2}$ An acronym for "A Verifier for Functional Programs".
} 
i.e. call-by-value. The use of incomplete conditionals like for redc and redc* results in incompletely defined procedures [12]. Such a feature is required when working with polymorphic data structures but is useful for monomorphic data structures too as it avoids the need for stipulating artificial results, e.g. for $n / 0$. Predicates are defined by procedures with result type bool. Procedure function $[$ infix $]>(x, y: \mathbb{N}):$ bool $<=\ldots$ for deciding the greater-than relation is the only predefined procedure in the system. Upon the definition of a procedure, VeriFun's automated termination analysis (based on the method of ArgumentBounded Functions $[8,11]$ ) is invoked for generating termination hypotheses which are sufficient for the procedure's termination and proved like lemmas. Afterwards induction axioms are computed from the terminating procedures' recursion structure to be on stock for future use.

Lemmas are defined with conditionals if : bool $\times$ bool $\times$ bool $\rightarrow$ bool as the main connective, but negation $\neg$ and case-conditionals may be used as well. Only universal quantification is allowed for the variables of a lemma. Figure 2 displays a lemma about (the elsewhere defined) procedure mod (computing the remainder function) which is frequently used in subsequent proofs. The string in the headline (between "lemma" and " $<=$ ") is just an identifier assigning a name to the lemma for reference and must not be confused with the statement of the lemma given as a boolean term in the lemma body. Some basic lemmas about equality and $>$, e.g. stating transitivity of $=$ and $>$, are predefined in the system. Predefined lemmas are frequently used in almost every case study so that work is eased by having them always available instead of importing them from some proof library.

Lemmas are proved with the HPL-calculus (abbreviating Hypotheses, Programs and Lemmas) [10]. The most relevant proof rules of this calculus are Induction, Use Lemma, Apply Equation, Unfold Procedure, Case Analysis and Simplification. Formulas are given as sequents of form $H, I H \vdash$ goal, where $H$ is a finite set of hypotheses given as literals, i.e. negated or unnegated predicate calls and equations, $I H$ is a finite set of induction hypotheses given as partially quantified boolean terms and goal is a boolean term, called the goalterm of the sequent. A deduction in the HPL -calculus is represented by a tree whose nodes are given by sequents. A lemma $\ell$ with body $\forall \ldots$ goal is verified iff $(i)$ the goalterm of each sequent at a leaf of the proof tree rooted in \{\}$,\{\} \vdash$ goal equals true and (ii) each lemma applied by Use Lemma or Apply Equation when building the proof tree is verified. The base of this recursive definition is given by lemmas being proved without using other lemmas. Induction hypotheses are treated like verified lemmas, however being available only in the sequent they belong to.

The Induction rule creates the base and step cases for a lemma from an induction axiom. By choosing Simplification, the system's first-order theorem prover, called the Symbolic Evaluator, is started for rewriting a sequent's goalterm using the hypotheses and induction hypotheses of the sequent, the definitions of the data structures and procedures as well as the lemmas already verified. This reasoner is guided by heuristics, e.g. for deciding whether to use a procedure definition, for speeding up proof search by filtering out useless lemmas, 
etc. Equality reasoning is implemented by conditional term rewriting with $A C$ matching, where the orientation of equations is heuristically established [13]. The Symbolic Evaluator is a fully automatic tool over which the user has no control, thus leaving the $H P L$-proof rules as the only means to guide the system to a proof.

Also the $H P L$-calculus is controlled by heuristics. When applying the Verify command to a lemma, the system starts to compute a proof tree by choosing appropriate $H P L$-proof rules heuristically. If a proof attempt gets stuck, the user must step in by applying a proof rule to some leaf of the proof tree (sometimes after pruning some unwanted branch of the tree), and the system then takes over control again. Also it may happen that a further lemma must be formulated by the user before the proof under consideration can be completed. All interactions are menu driven so that typing in proof scripts is avoided (see $[7,10])$.

Verifun is implemented in JAVA and installers for running the system under Windows, Unix/Linux or Mac are available from the web [7]. When working with the system, we use proof libraries which had been set up over the years by extending them with definitions and lemmas being of general interest. When importing a definition or a lemma from a library into a case study, all program elements and proofs the imported item depends on are imported as well. The correctness proofs for Montgomery Multiplication depend on 9 procedures and 96 lemmas from our arithmetic proof library, which ranges from simple statements like associativity and commutativity of addition up to more ambitious theorems about primes and modular arithmetic. In the sequel we will only list the lemmas which are essential to understand the proofs and refer to [7] for a complete account of all used lemmas and their proofs.

\section{Multiplicative Inverses}

We start our development by stipulating how multiplicative inverses are computed. To this effect we have to define some procedure $\mathfrak{I}: \mathbb{N} \times \mathbb{N} \rightarrow \mathbb{N}$ satisfying ${ }^{3}$

$$
\begin{gathered}
\forall x, y: \mathbb{N} y \neq 0 \wedge \operatorname{gcd}(x, y)=1 \rightarrow[x \cdot \mathfrak{I}(x, y) \equiv 1] \bmod y \\
\forall x, y, z: \mathbb{N} y \neq 0 \wedge \operatorname{gcd}(x, y)=1 \rightarrow[z \cdot x \cdot \Im(x, y) \equiv z] \bmod y \\
\forall n, x, y, z: \mathbb{N} y \neq 0 \wedge \operatorname{gcd}(x, y)=1 \rightarrow[n+z \cdot x \cdot \mathfrak{I}(x, y) \equiv n+z] \bmod y
\end{gathered}
$$

Lemma 2 is proved with Lemma 1 and library lemma

$$
\forall n, m, x, y: \mathbb{N} \operatorname{gcd}(n, m)=1 \wedge[m \cdot x \equiv m \cdot y] \bmod n \rightarrow[x \equiv y] \bmod n
$$

after instructing the system to use library lemma

$$
\forall x, y, z: \mathbb{N} z \neq 0 \rightarrow[x \cdot(y \bmod z) \equiv x \cdot y] \bmod z
$$

$\overline{{ }^{3} \text { If } x, y, z \in \mathbb{Z}}$ and $n \in \mathbb{N}$, then $n \mid z$ abbreviates $z \bmod n=0$, where $z \bmod n=-(|z|$ $\bmod n)$ if $z<0$, and $[x \equiv y] \bmod n$ stands for $n \mid x-y \cdot x \bmod n=y \bmod n$ is sufficient for $[x \equiv y] \bmod n$ but only necessary, if $x$ and $y$ have same polarity. 
and $\sqrt{ }$ erifun proves Lemma 3 automatically using Lemma 2 as well as library lemma

$$
\forall n, x, y, z: \mathbb{N} z \neq 0 \wedge[x \equiv y] \bmod z \rightarrow[x+n \equiv y+n] \bmod z .
$$

Multiplicative inverses can be computed straightforwardly with Euler's $\phi$ function, where Lemma 1 then is proved with Euler's Theorem [7,14]. But this approach is very costly and therefore unsuitable for an implementation of Montgomery Multiplication.

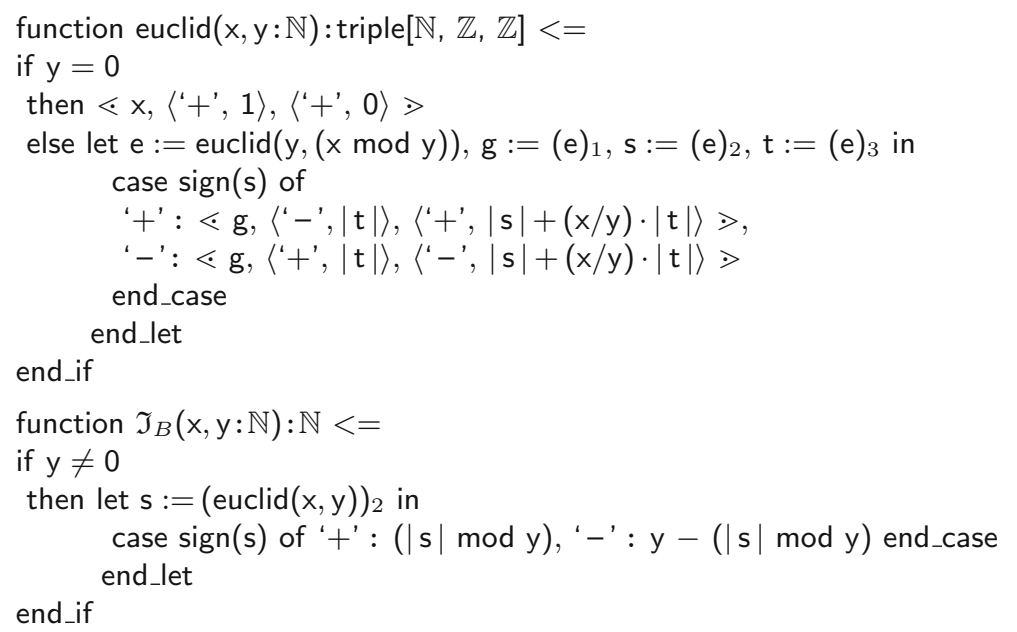

Fig. 3. Computation of multiplicative inverses by the extended Euclidean algorithm

\subsection{Bézout's Lemma}

A more efficient implementation of procedure $\mathfrak{I}$ is based on Bézout's Lemma stating that the greatest common divisor can be represented as a linear combination of its arguments:

\section{Bézout's Lemma}

For all $x, y \in \mathbb{N}$ some $s, t \in \mathbb{Z}$ exist such that $\operatorname{gcd}(x, y)=x \cdot s+y \cdot t$.

If $y \neq 0, \mathfrak{I}_{B}(x, y):=s \bmod y$ is defined and $\operatorname{gcd}(x, y)=1$ holds, then by Bézout's Lemma $\left[x \cdot \mathfrak{I}_{B}(x, y)=x \cdot(s \bmod y) \equiv x \cdot s \equiv x \cdot s+y \cdot t=1\right] \bmod y$. To implement this approach, the integer $s$ need to be computed which can be performed by the extended Euclidean algorithm displayed in Fig. 3. This approach is more efficient because a call of $\operatorname{euclid}(x, y)$ (and in turn of $\mathfrak{I}_{B}(x, y)$ given as in Fig. 3) can be computed in time proportional to $(\log y)^{2}$ if $x<y$, whereas the use of Euler's $\phi$-function needs time proportional to $2^{\log y}$ in the context of Montgomery Multiplication (as $\left.\phi\left(2^{k+1}\right)=2^{k}\right)$.

However, $s \in \mathbb{Z}$ might be negative so that $y+(s \bmod y) \in \mathbb{N}$ instead of $s \bmod y$ then must be used as the multiplicative inverse of $x$ because the carriers 


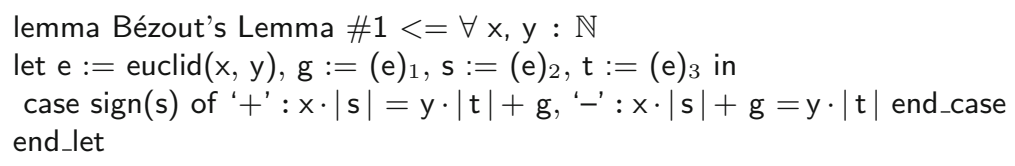

lemma Bézout's Lemma $\# 2<=\forall x, y: \mathbb{N}(\operatorname{euclid}(x, y))_{1}=\operatorname{gcd}(x, y)$.

Fig. 4. Bézout's Lemma

of the rings $R_{n}$ and $R_{n}^{m}$ are subsets of $\mathbb{N}$. We therefore define $\mathfrak{I}_{B}$ as shown in Fig. 3 which complicates the proof of Lemma 1 (with $\mathfrak{I}$ replaced by $\mathfrak{I}_{B}$ ) as this definition necessitates a proof of $[x \cdot y+x \cdot(s \bmod y) \equiv 1] \bmod y$ if $s<0$.

Bézout's Lemma is formulated in our system's notation by the pair of lemmas displayed in Fig. 4. When prompted to prove Lemma 7, the system starts a Peano induction upon $x$ but gets stuck in the step case. We therefore command to use induction corresponding to the recursion structure of procedure euclid. VeriFun responds by proving the base case and simplifying the induction conclusion in case $\operatorname{sign}(s)=$ ' + ' to

$y \neq 0 \rightarrow x \cdot|t|+g=(x \bmod y) \cdot|t|+g+|t| \cdot(y-1) \cdot(x / y)+|t| \cdot(x / y)$

(where $e$ abbreviates $\operatorname{euclid}(y,(x \bmod y)), g:=(e)_{1}, s:=(e)_{2}$ and $\left.t:=(e)_{3}\right)$ using the induction hypothesis

$$
\begin{aligned}
\forall x^{\prime}: \mathbb{N} \operatorname{let}\{e & :=\operatorname{euclid}\left(x^{\prime},(x \bmod y)\right), g:=(e)_{1}, s:=(e)_{2}, t:=(e)_{3} \\
\operatorname{case}\{\operatorname{sign}(s) ; & \\
& \text { '+' }: x^{\prime} \cdot|s|=(x \bmod y) \cdot|t|+g \\
& \text { '-' } \left.\left.: x^{\prime} \cdot|s|+g=(x \bmod y) \cdot|t|\right\}\right\}
\end{aligned}
$$

and some basic arithmetic properties. We then instruct the system to use the quotient-remainder theorem for replacing $x$ at the left-hand side of the equation in (i) by $(x / y) \cdot y+(x \bmod y)$ causing $\sqrt{ }$ eriFun to complete the proof. The system computes a similar proof obligation for case $\operatorname{sign}(s)={ }^{\text {' }}$ ' which is proved in the same way.

By "basic arithmetic properties" we mean well known facts like associativity, commutativity, distributivity, cancellation properties etc. of $+,-, \cdot, /, g c d, \ldots$ which are defined and proved in our arithmetic proof library. These facts are used almost everywhere by the Symbolic Evaluator so that we will not mention their use explicitly in the sequel.

When called to prove Lemma 8 by induction corresponding to the recursion structure of procedure euclid, VeriFun responds by proving the base case and rewrites the step case with the induction hypothesis to

$$
y \neq 0 \rightarrow \operatorname{gcd}(x, y)=\operatorname{gcd}(y,(x \bmod y)) .
$$


It then automatically continues with proving (ii) by induction corresponding to the recursion structure of procedure gcd where it succeeds for the base and the step case. Lemma 8 is useful because it relates procedure euclid to procedure gcd of our arithmetic proof library so that all lemmas about gcd can be utilized for the current proofs.

For proving the inverse property

$$
\forall x, y: \mathbb{N} y \neq 0 \wedge \operatorname{gcd}(x, y)=1 \rightarrow\left[x \cdot \mathfrak{I}_{B}(x, y) \equiv 1\right] \bmod y
$$

of procedure $\mathfrak{I}_{B}$, we call the system to unfold procedure call $\mathfrak{I}_{B}(x, y)$. $\sqrt{\text { eriFun }}$ responds by proving the statement for case $\operatorname{sign}(s)={ }^{+}+$' using Bézout's Lemma 7 and 8 and the library lemmas

$$
\begin{gathered}
\forall x, y, z: \mathbb{N} z \neq 0 \wedge z \mid x \rightarrow[x+y \equiv y] \bmod z \\
\forall x, y: \mathbb{N} y \neq 0 \rightarrow y \mid x \cdot y
\end{gathered}
$$

as well as (5), but gets stuck in the remaining case with proof obligation

$$
y \neq 0 \wedge \operatorname{sign}(s)='-' \wedge g=1 \rightarrow[x \cdot y-x \cdot(|s| \bmod y) \equiv 1] \bmod y
$$

where $g$ abbreviates $(\operatorname{euclid}(x, y))_{1}$ and $s$ stands for $(\operatorname{euclid}(x, y))_{2}$. Proof obligation (iii) represents the unpleasant case of the proof development and necessitates the invention of an auxiliary lemma for completing the proof. After some unsuccessful attempts, we eventually came up with lemma

$$
\forall x, y, z, u: \mathbb{N} y \neq 0 \wedge y \mid(x \cdot z+u) \wedge x \geq u \rightarrow[x \cdot y-x \cdot(z \bmod y) \equiv u] \bmod y .
$$

For proving (iii), we command to use Lemma 12 for replacing the left-hand side of the congruence in (iii) by $g$, and $\sqrt{ }$ eriFun computes

$$
\begin{aligned}
& y \neq 0 \wedge \operatorname{sign}(s)=\cdot-' \wedge g=1 \rightarrow \\
& \quad(x \geq g \rightarrow y \mid(x \cdot|s|+g)) \wedge \\
& \quad(x<g \rightarrow[x \cdot y-x \cdot(|s| \bmod y) \equiv 1] \bmod y .
\end{aligned}
$$

Now we can call the system to use Bézout's Lemma 7 for replacing $x \cdot|s|+g$ in (iv) by $y \cdot|t|$ causing $\sqrt{ }$ eriFun to complete the proof with Bézout's Lemma 8 and library lemma (11) in case of $x \geq g$ and otherwise showing that $x<g=1$ entails $x=0$ and $1=g=\operatorname{gcd}(0, y)=y$ in turn, so that $x \cdot y-x \cdot(|s| \bmod y)$ simplifies to 0 and $[0 \equiv 1] \bmod y$ rewrites to true.

It remains to prove auxiliary lemma (12) for completing the proof of Lemma 9: After being called to use library lemma ${ }^{4}$

$$
\forall x, y, z: \mathbb{N} z \neq 0 \wedge z|(x-y) \wedge z|(y-x) \rightarrow[x \equiv y] \bmod z
$$

\footnotetext{
${ }^{4}$ At least one of $z \mid(x-y)$ or $z \mid(y-x)$ holds trivially because subtraction is defined such that $a-b=0$ iff $a \leq b$.
} 
for replacing the left-hand side of the congruence in (12) by $u$, VeriFun computes

$$
y \neq 0 \wedge y|(x \cdot z+u) \wedge x \geq u \rightarrow y|(u-(x \cdot y-x \cdot(z \bmod y)))
$$

with the library lemmas (11) and

$$
\begin{gathered}
\forall x, y, z: \mathbb{N} z \neq 0 \wedge[x \equiv y] \bmod z \rightarrow z \mid(x-y) \\
\forall x, y, z, n: \mathbb{N} n \neq 0 \rightarrow[x+y \cdot(z \bmod n) \equiv x+y \cdot z] \bmod n .
\end{gathered}
$$

We then command to use library lemma $\forall x, y, z: \mathbb{N} z \neq 0 \wedge x \leq y \rightarrow x \leq y \cdot z$ (with $u$ substituted for $x, x$ for $y$ and $y-(z \bmod y)$ for $z$ ) after $x$ factoring out, causing $\sqrt{ }$ erifun to prove $(\mathrm{v})$ with the synthesized lemma ${ }^{5}$

$$
\forall x, y: \mathbb{N} y \neq 0 \rightarrow y>(x \bmod y) .
$$

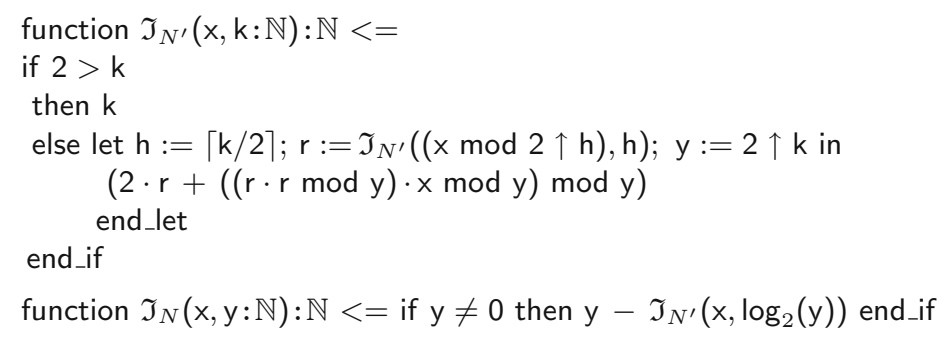

Fig. 5. Computation of multiplicative inverses by Newton-Raphson iteration

\subsection{Newton's Method}

Newton-Raphson iteration is a major tool in arbitrary-precision arithmetic and efficient algorithms for computing multiplicative inverses are developed in combination with Hensel Lifting [2]. Figure 5 displays an implementation by procedure $\mathfrak{I}_{N}$ for odd numbers $x$ and powers $y$ of 2 (where $\uparrow$ computes exponentiation satisfying $0 \uparrow 0=1$ ). Procedure $\mathfrak{I}_{N}$ is defined via procedure $\mathfrak{I}_{N^{\prime}}$ which is obtained from [3], viz. Algorithm 2' Recursive Hensel, where however '-' instead of ' + ' is used in the result term. Algorithm 2 ' was developed to compute a multiplicative inverse of $x$ modulo $p^{k}$ for any $x$ not dividable by a prime $p$ and returns a negative integer in most cases. By replacing '-' with '+', all calculations can be kept within $\mathbb{N}$ so that integer arithmetic is avoided. As procedure $\mathfrak{I}_{N^{\prime}}$ computes the absolute value of a negative integer computed by Algorithm $2^{\prime}$, one additional subtraction is needed to obtain a multiplicative inverse which is implemented by procedure $\mathfrak{I}_{N}$. The computation of $\mathfrak{I}_{N}\left(x, 2^{k}\right)$ only requires $\log k$ steps (compared to $k^{2}$ steps for $\mathfrak{I}_{B}\left(x, 2^{k}\right)$ ), and therefore $\mathfrak{I}_{N}$ is the method of choice for computing a Montgomery Inverse.

\footnotetext{
${ }^{5}$ Synthesized lemmas are a spin-off of the system's termination analysis.
} 
However, Algorithm $2^{\prime}$ is flawed so that we wasted some time with our verification attempts: The four mod-calls in the algorithm are not needed for correctness, but care for efficiency as they keep the intermediate numbers small. Now instead of using modulus $2^{k}$ for both inner mod-calls, Algorithm $2^{\prime}$ calculates $\bmod 2^{\lceil k / 2\rceil}$ thus spoiling correctness. As the flawed algorithm cares for even smaller numbers, the use of $\bmod 2^{\lceil k / 2\rceil}$ could be beneficial indeed, and therefore it was not obvious to us whether we failed in the verification only because some mathematical argumentation was missing. But this consideration put us on the wrong track. Becoming eventually frustrated by the unsuccessful verification attempts, we started VeriFun's Disprover [1] which — to our surprise - came up with the counter example $x=3, k=2$ for Lemma 17 in less than a second. ${ }^{6}$ We then repaired the algorithm as displayed in Fig. 5 and subsequently verified it (cf. Lemma 20). Later we learned that the fault in Algorithm $2^{\prime}$ has not been recognized so far and that one cannot do better to patch it as we did. ${ }^{7}$

For proving the inverse property (20) of procedure $\mathfrak{I}_{N}$, we first have to verify the correctness statement

$$
\forall x, k: \mathbb{N} 2 \nmid x \rightarrow\left(x \cdot \mathfrak{I}_{N^{\prime}}(x, k) \bmod 2^{k}\right)=2^{k}-1
$$

for procedure $\mathfrak{I}_{N^{\prime}}$ : We call the system to use induction corresponding to the recursion structure of procedure $\mathfrak{I}_{N^{\prime}}$ which provides the induction hypothesis

$$
\forall x^{\prime}: \mathbb{N} k \geq 2 \wedge 2 \nmid x^{\prime} \rightarrow\left(x^{\prime} \cdot \mathfrak{I}_{N^{\prime}}\left(x^{\prime},\lceil k / 2\rceil\right) \bmod 2^{\lceil k / 2\rceil}\right)=2^{\lceil k / 2\rceil}-1 .
$$

eriFun proves the base case, but gets stuck in the step case with

$$
\begin{aligned}
k \geq 2 & \wedge 2 \nmid x \rightarrow \\
& \left(x \cdot\left(2 A+\left(x \cdot\left(A^{2} \bmod 2^{k}\right) \bmod 2^{k}\right) \bmod 2^{k}\right) \bmod 2^{k}\right)=2^{k}-1
\end{aligned}
$$

where $A$ stands for $\mathfrak{I}_{N^{\prime}}\left(\left(x \bmod 2^{\lceil k / 2\rceil}\right),\lceil k / 2\rceil\right)$. By prompting the system to use Lemma 5, proof obligation (i) is simplified to

$$
k \geq 2 \wedge 2 \nmid x \rightarrow\left(2 B+B^{2} \bmod 2^{k}\right)=2^{k}-1
$$

(where $B$ abbreviates $x \cdot A$ ) thus eliminating the formal clutter resulting from the mod-calls in procedure $\mathfrak{I}_{N^{\prime}}$. Next we replace $2 B+B^{2}$ by $(B+1)^{2}-1$ and then call the system to replace $B$ by $(B / C) \cdot C+R$ where $C=2^{\lceil k / 2\rceil}$ and $R=$ $((x \bmod C) \cdot A \bmod C)$, which is justified by the quotient-remainder theorem as $R$ rewrites to $(B \bmod C)$ by library lemma $(5)$. This results in proof obligation

$$
k \geq 2 \wedge 2 \nmid x \rightarrow\left(((B / C) \cdot C+R+1)^{2}-1 \bmod 2^{k}\right)=2^{k}-1
$$

\footnotetext{
${ }^{6}$ The Disprover is based on two heuristically controlled disproving calculi, and its implementation provides four selectable execution modes (Fast Search, Extended Search, Simple Terms and Structure Expansion). For difficult problems, the user may support the search for counter examples by presetting some of the universally quantified variables with general terms or concrete values.

7 Personal communication with Jean-Guillaume Dumas.
} 
and we command to use the induction hypothesis (18) for replacing $R$ in (iii) by $C-1$. Verifun then responds by computing

$$
k \geq 2 \wedge 2 \nmid x \rightarrow\left(((B / C) \cdot C+C)^{2}-1 \bmod 2^{k}\right)=2^{k}-1
$$

using library lemmas $\forall x, y, z: \mathbb{N} y \neq 0 \wedge z \neq 0 \wedge z \mid y \rightarrow[(x \bmod y) \equiv x] \bmod z$ and (5) to prove $2 \nmid\left(x \bmod 2^{\lceil k / 2\rceil}\right)$ for justifying the use of the induction hypothesis. When instructed to factor out $C$ in (iv), the system computes

$$
k \geq 2 \wedge 2 \nmid x \rightarrow\left(\left(2^{\lceil k / 2\rceil}\right)^{2} \cdot(B / C+1)^{2}-1 \bmod 2^{k}\right)=2^{k}-1 .
$$

We command to use library lemma

$$
\forall x, y, z: \mathbb{N} z \neq 0 \wedge z \nmid x \wedge z \mid y \wedge y \geq x \rightarrow(y-x \bmod z)=z-(x \bmod z)
$$

for replacing the left-hand side of the equation in $(\mathrm{v})$ yielding

$$
k \geq 2 \wedge 2 \nmid x \rightarrow 2^{k}-\left(1 \bmod 2^{k}\right)=2^{k}-1
$$

justified by proof obligation

$$
\begin{aligned}
k \geq 2 & \wedge 2 \nmid x \rightarrow \\
2^{k} & \neq 0 \wedge 2^{k} \nmid 1 \wedge 2^{k} \mid\left(2^{\lceil k / 2\rceil}\right)^{2} \cdot(B / C+1)^{2} \wedge\left(2^{\lceil k / 2\rceil}\right)^{2} \cdot(B / C+1)^{2} \geq 1
\end{aligned}
$$

which $\sqrt{ }$ erifun simplifies to

$$
k \geq 2 \wedge 2 \nmid x \rightarrow 2^{k} \mid\left(2^{\lceil k / 2\rceil}\right)^{2} \cdot(B / C+1)^{2}
$$

in a first step. It then uses auxiliary lemma $\forall x: \mathbb{N} x \leq 2 \cdot\lceil x / 2\rceil$ and the library lemmas (11) and $\forall x, y, z: \mathbb{N} x \neq 0 \wedge z \leq y \rightarrow x^{z} \mid x^{y}$ for rewriting (vii) subsequently to true. Finally the system simplifies (vi) to true as well by unfolding the call of procedure mod, and Lemma 17 is proved.

When called to verify the inverse property

$$
\forall x, y: \mathbb{N} 2 \nmid x \wedge 2^{?}(y) \rightarrow\left[x \cdot \mathfrak{I}_{N}(x, y) \equiv 1\right] \bmod y
$$

of procedure $\mathfrak{I}_{N}$ (where $2^{?}(y)$ decides whether $y$ is a power of 2 ), $\sqrt{ }$ eriFun unfolds the call of procedure $\mathfrak{I}_{N}$ and returns

$$
y \geq 2 \wedge 2 \nmid x \wedge 2^{?}(y) \rightarrow\left(x \cdot y-x \cdot \mathfrak{I}_{N^{\prime}}\left(x, \log _{2}(y)\right) \bmod y\right)=1 .
$$

Now we instruct the system to use library lemma (19) for replacing the left-hand side of the equation in (viii), and VeriFun computes

$$
\begin{aligned}
& y \geq 2 \wedge 2 \nmid x \wedge 2^{?}(y) \rightarrow \\
& \quad\left(x \cdot \mathfrak{I}_{N^{\prime}}\left(x, \log _{2}(y)\right) \bmod y\right) \neq 0 \wedge y-\left(x \cdot \mathfrak{I}_{N^{\prime}}\left(x, \log _{2}(y)\right) \bmod y\right)=1
\end{aligned}
$$

using auxiliary lemma $\forall x, y: \mathbb{N} 2^{?}(y) \rightarrow y>\mathfrak{I}_{N^{\prime}}\left(x, \log _{2}(y)\right)$ and the library lemmas (11), (14) and

$$
\forall x, y, z: \mathbb{N} x \cdot y>x \cdot z \rightarrow y>z .
$$

Finally we let the system use library lemma $\forall x: \mathbb{N} 2^{?}(x) \rightarrow 2^{\log _{2}(x)}=x$ to replace both moduli $y$ in (ix) by $2^{\log _{2}(y)}$ causing VeriFun to rewrite both occurrences of $\left(x \cdot \mathfrak{I}_{N^{\prime}}\left(x, \log _{2}(y)\right) \bmod y\right)$ with Lemma 17 to $y-1$ and proof obligation (ix) to true in turn, thus completing the proof of (20). 


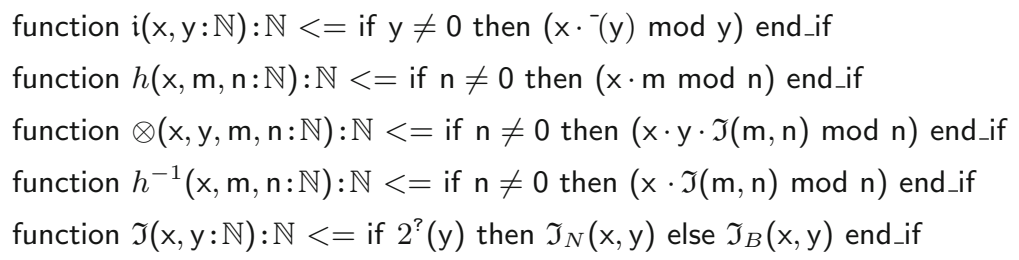

Fig. 6. Procedures for verifying Montgomery Multiplication

\section{Correctness of Montgomery Multiplication}

We continue by defining procedures for computing the functions $\mathfrak{i}, h, \otimes$ and $h^{-1}$ as displayed in Fig. 6 , where we write $\mathfrak{i}(x, y)$ instead of $\mathfrak{i}_{y}(x)$ in the procedures and lemmas. As we aim to prove correctness of Montgomery Multiplication using procedure $\mathfrak{I}_{N}$ for computing the Montgomery Inverse with minimal costs, $2 \nmid n \wedge 2^{?}(m)$ instead of $\operatorname{gcd}(n, m)=1$ must be demanded to enable the use of Lemma 20 when proving the statements of Theorems 1 and 2. However, the multiplicative inverses $n_{m}^{-1}$ and $m_{n}^{-1}$ both are needed in the proofs (whereas only $n_{m}^{-1}$ is used in applications of redc and redc*). Consequently procedure $\mathfrak{I}_{N}$ cannot be used in the proofs as it obviously fails in computing $m_{n}^{-1}$ (except for case $n=m=1$, of course). This problem does not arise if procedure $\mathfrak{I}_{B}$ is used instead, where $\operatorname{gcd}(n, m)=1$ is demanded, because $\mathfrak{I}_{B}(n, m)=n_{m}^{-1}$ and $\mathfrak{I}_{B}(m, n)=m_{n}^{-1}$ for any coprimes $n$ and $m$ by Lemma 9 . The replacement of $\mathfrak{I}_{B}$ by $\mathfrak{I}_{N}$ when computing the Montgomery Inverse then must be justified afterwards by additionally proving

$$
\forall x, y: \mathbb{N} 2 \nmid x \wedge 2^{?}(y) \rightarrow \mathfrak{I}_{B}(x, y)=\mathfrak{I}_{N}(x, y) .
$$

However, proving (22) would be a complicated and difficult enterprise because the recursion structures of procedures euclid and $\mathfrak{I}_{N^{\prime}}$ differ significantly. But we can overcome this obstacle by a simple workaround: We use procedure $\mathfrak{I}$ of Fig. 6 instead of $\mathfrak{I}_{B}$ in the proofs and let the system verify the inverse property

$$
\forall x, y: \mathbb{N} y \neq 0 \wedge \operatorname{gcd}(x, y)=1 \rightarrow[x \cdot \mathfrak{I}(x, y) \equiv 1] \bmod y
$$

of procedure $\mathfrak{I}$ before: $\sqrt{ }$ eriFun easily succeeds with library lemma (4) and the inverse property (9) of procedure $\mathfrak{I}_{B}$ after being instructed to use library lemma $\forall x, y, n: \mathbb{N} n \geq 2 \wedge n \mid y \wedge \operatorname{gcd}(x, y)=1 \rightarrow n \nmid x$ and the inverse property (20) of procedure $\mathfrak{I}_{N}$. Consequently $\mathfrak{I}(n, m)=n_{m}^{-1}$ and $\mathfrak{I}(m, n)=m_{n}^{-1}$ for any coprimes $n$ and $m$, and therefore $\mathfrak{I}$ can be used in the proofs. The use of $\mathfrak{I}_{N}$ instead of $\mathfrak{I}$ when computing the Montgomery Inverse is justified afterwards with lemma

$$
\forall x, y: \mathbb{N} 2^{?}(y) \rightarrow \mathfrak{I}(x, y)=\mathfrak{I}_{N}(x, y)
$$

having an obviously trivial (and automatic) proof. 
Central for the proofs of Theorems 1 and 2 is the key property

$$
\begin{aligned}
\forall m, n, x: \mathbb{N} \quad & m>n \wedge n \cdot m>x \wedge \operatorname{gcd}(n, m)=1 \rightarrow \\
& \operatorname{redc}(x, \mathfrak{i}(\Im(n, m), m), m, n)=(x \cdot \mathfrak{I}(m, n) \bmod n)
\end{aligned}
$$

of procedure redc: For proving Theorem 1.1

$$
\begin{aligned}
& \forall m, n, a: \mathbb{N} m>n>a \wedge \operatorname{gcd}(n, m)=1 \rightarrow \\
& \quad h(a, m, n)=\operatorname{redc}(a \cdot(m \cdot m \bmod n), \mathfrak{i}(\mathfrak{I}(n, m), m), m, n)
\end{aligned}
$$

we command to use (23) for replacing the right-hand side of the equation by $(a \cdot(m \cdot m \bmod n) \cdot \mathfrak{I}(m, n) \bmod n)$. The system then replaces the left-hand side of the equation with $a \cdot m \bmod n$ by unfolding procedure call $h(a, m, n)$ and simplifies the resulting equation to true with Lemma 2, the synthesized lemma (16) and the library lemmas (5) and

$$
\forall x, y, u, v: \mathbb{N} x>y \wedge u>v \rightarrow x \cdot u>y \cdot v .
$$

Theorems 1.2 and 1.3, viz.

$$
\begin{aligned}
& \forall m, n, a, b: \mathbb{N} m>n>a \wedge n>b \wedge g c d(n, m)=1 \\
& \rightarrow \otimes(a, b, m, n)=\operatorname{redc}(a \cdot b, \mathfrak{i}(\mathfrak{I}(n, m), m), m, n) \\
& \forall m, n, a: \mathbb{N} m>n>a \wedge \operatorname{gcd}(n, m)=1 \\
& \rightarrow h^{-1}(a, m, n)=\operatorname{redc}(a, \mathfrak{i}(\mathfrak{I}(n, m), m), m, n)
\end{aligned}
$$

are (automatically) proved in the same way.

Having proved Theorem 1, it remains to verify the key property (23) for procedure redc (before we consider Theorem 2 subsequently). We start by proving that division by $m$ in $R_{n}$ can be expressed by $\mathfrak{I}$ : We call the system to prove

$$
\forall m, n, x: \mathbb{N} n \neq 0 \wedge m \mid x \wedge \operatorname{gcd}(n, m)=1 \rightarrow[x / m \equiv x \cdot \Im(m, n)] \bmod n
$$

and $\sqrt{ }$ erifun automatically succeeds with Lemma 2 and the library lemmas (4) and $\forall x, y, z: \mathbb{N} y \neq 0 \wedge y \mid x \rightarrow(x / y) \cdot y=x$.

As a consequence of Lemma 25, the quotient $q$ in procedure redc can be expressed in $R_{n}$ by $\mathfrak{I}$ in particular (if redc is called with the Montgomery Inverse as actual parameter for the formal parameter $z$ ), which is stated by lemma

$$
\begin{aligned}
& \forall m, n, x: \mathbb{N} n \neq 0 \wedge \operatorname{gcd}(n, m)=1 \\
& \quad \rightarrow[(x+n \cdot(x \cdot \mathfrak{i}(\mathfrak{I}(n, m), m) \bmod m)) / m \equiv x \cdot \mathfrak{I}(m, n)] \bmod n
\end{aligned}
$$

For obtaining a proof, we command to use Lemma 25 for replacing the left-hand side of the congruence in $(26)$ by $(x+n \cdot(x \cdot \mathfrak{i}(\mathfrak{I}(n, m), m) \bmod m)) \cdot \mathfrak{I}(m, n)$ 
causing $\sqrt{ }$ eriFun to complete the proof using Lemma 3 as well as the library lemmas (5), (10), (11), (15) and $\forall x, y: \mathbb{N} y \neq 0 \rightarrow y \mid(x+(y-1) \cdot x)$.

An obvious correctness demand for the method is that each call of redc (under the given requirements) computes some element of the residue class $\bmod n$. This is guaranteed by the conditional subtraction of $n$ from the quotient $q$ in the body of procedure redc. However, at most one subtraction of $n$ from $q$ results in the desired property only if $n+n>q$ holds, which is formulated by lemma

$$
\forall m, n, x: \mathbb{N} m \cdot n>x \rightarrow n+n>(x+n \cdot(x \cdot \mathfrak{i}(\mathfrak{I}(n, m), m) \bmod m)) / m .
$$

We prompt the system to use a case analysis upon $m \cdot(n+n)>x+n \cdot(x$. $\mathfrak{i}(\mathfrak{I}(n, m), m) \bmod m)$ causing $\sqrt{ }$ erifun to prove the statement in the positive case with the library lemmas (5) and $\forall x, y, z: \mathbb{N} x \cdot z>y \rightarrow x>y / z$ and to verify it in the negative case with the synthesized lemma (16) and the library lemmas (5), (21) and $\forall x, y, u, v: \mathbb{N} x>y \wedge u \geq v \rightarrow x+u>y+v$.

Now the $\bmod n$ property of procedure redc can be verified by proving lemma

$$
\begin{aligned}
& \forall m, n, x: \mathbb{N} m>n \wedge n \cdot m>x \wedge \operatorname{gcd}(n, m)=1 \rightarrow \\
& \quad \operatorname{red} c(x, \mathfrak{i}(\Im(n, m), m), m, n)=(\operatorname{red}(x, \mathfrak{i}(\mathfrak{I}(n, m), m), m, n) \bmod n) .
\end{aligned}
$$

We let the system unfold the call of procedure mod in (28) causing VeriFun to use the synthesized lemma (16) for computing the simplified proof obligation

$$
m>n \wedge n \cdot m>x \wedge \operatorname{gcd}(n, m)=1 \rightarrow n>\operatorname{redc}(x, \mathfrak{i}(\mathfrak{I}(n, m), m), m, n) .
$$

Then we command to unfold the call of procedure redc which simplifies to

$$
\begin{aligned}
& m>n \wedge n \cdot m>x \wedge \operatorname{gcd}(n, m)=1 \wedge \\
&(x+n \cdot(x \cdot \mathfrak{i}(\mathfrak{I}(n, m), m) \bmod m)) / m \geq n \\
& \quad \rightarrow n>(x+n \cdot(x \cdot \mathfrak{i}(\mathfrak{I}(n, m), m) \bmod m)) / m-n .
\end{aligned}
$$

Finally we let the system use library lemma $\forall x, y, z: \mathbb{N} x>y \wedge y \geq z \rightarrow x-z>$ $y-z$ resulting in proof obligation

$$
\begin{aligned}
& m>n \wedge n \cdot m>x \wedge \operatorname{gcd}(n, m)=1 \\
& \wedge(x+n \cdot(x \cdot \mathfrak{i}(\mathfrak{I}(n, m), m) \bmod m)) / m \geq n \\
& {[n+n>(x+n \cdot(x \cdot \mathfrak{i}(\mathfrak{I}(n, m), m) \bmod m)) / m \wedge} \\
& \wedge(x+n \cdot(x \cdot \mathfrak{i}(\mathfrak{I}(n, m), m) \bmod m)) / m \geq n \\
& \rightarrow(n+n)-n>(x+n \cdot(x \cdot \mathfrak{i}(\mathfrak{I}(n, m), m) \bmod m)) / m-n] \\
& \rightarrow n>(x+n \cdot(x \cdot \mathfrak{i}(\mathfrak{I}(n, m), m) \bmod m)) / m-n
\end{aligned}
$$

which simplifies to

$$
\begin{aligned}
& m>n \wedge n \cdot m>x \wedge \operatorname{gcd}(n, m)=1 \\
& \wedge(x+n \cdot(x \cdot \mathfrak{i}(\mathfrak{I}(n, m), m) \bmod m)) / m \geq n \\
& \wedge(n+n)-n>(x+n \cdot(x \cdot \mathfrak{i}(\mathfrak{I}(n, m), m) \bmod m)) / m-n \\
& \rightarrow n>(x+n \cdot(x \cdot \mathfrak{i}(\Im(n, m), m) \bmod m)) / m-n
\end{aligned}
$$

by Lemma 27 and to true in turn using the plus-minus cancellation. 
Now all lemmas for proving the key lemma (23) are available: We demand to use Lemma 28 for replacing the left-hand side of the equation in (23) by $(\operatorname{red} c(x, \mathfrak{i}(\mathfrak{I}(n, m), m), m, n) \bmod n)$ and to apply lemma $(26)$ for replacing the right-hand side by $((x+n \cdot(x \cdot \mathfrak{i}(\mathfrak{I}(n, m), m) \bmod m)) / \operatorname{m} \bmod n)$ resulting in the simplified proof obligation

$$
\begin{aligned}
& m>n \wedge n \cdot m>x \wedge \operatorname{gcd}(n, m)=1 \rightarrow \\
& \quad[\operatorname{redc}(x, \mathfrak{i}(\Im(n, m), m), m, n) \equiv(x+n \cdot(x \cdot \mathfrak{i}(\mathfrak{I}(n, m), m) \bmod m)) / m] \bmod n .
\end{aligned}
$$

Then we unfold the call of procedure redc causing the system to prove (v) with library lemma (5).

Having proved the key lemma (23), the proof of Theorem 2

$$
\begin{aligned}
& \forall m, n, a, j: \mathbb{N} m>n>a \wedge \operatorname{gcd}(n, m)=1 \rightarrow \\
& \quad\left(a^{j} \bmod n\right)=\operatorname{redc}\left(\operatorname{redc} c^{*}(\operatorname{redc}(a \cdot M, I, m, n), I, m, n, j), I, m, n\right)
\end{aligned}
$$

(where $M=((m \cdot m) \bmod n)$ and $I=\mathfrak{i}(\mathfrak{I}(n, m), m))$ is easily obtained by support of a further lemma, viz.

$$
\begin{aligned}
& \forall m, n, a, j: \mathbb{N} m>n>a \wedge \operatorname{gcd}(n, m)=1 \rightarrow \\
& \quad\left(m \cdot a^{j} \bmod n\right)=\operatorname{redc} c^{*}(\operatorname{redc}(a \cdot M, I, m, n), I, m, n, j) .
\end{aligned}
$$

When called to use Peano induction upon $j$ for proving (29), $\sqrt{ }$ eriFun proves the base case and rewrites the step case with the induction hypothesis to

$$
\begin{aligned}
& m>n>a \wedge \operatorname{gcd}(n, m)=1 \wedge j \neq 0 \rightarrow \\
& \left(m \cdot a^{j-1} \cdot a \bmod n\right)=\operatorname{redc}\left(\operatorname{redc}(a \cdot M, I, m, n) \cdot\left(m \cdot a^{j-1} \bmod n\right), I, m, n\right) .
\end{aligned}
$$

Then we command to replace both calls of redc with the key lemma (23) causing Verifun to succeed with the lemmas (2), (5), (16) and (24).

Finally the system proves (Thm 2) using lemmas (2), (5), (16), (29) and library lemma $\forall x, y, z: \mathbb{N} x \neq 0 \wedge y>z \rightarrow x \cdot y>z$ after being prompted to use (Thm 1.3) for replacing the right-hand side of the equation in (Thm 2).

\section{Discussion and Conclusion}

We presented machine assisted proofs verifying an efficient implementation of Montgomery Multiplication, where we developed the proofs ourselves as we are not aware of respective proofs published elsewhere. Our work also uncovered a serious fault in a published algorithm for computing multiplicative inverses based on Newton-Raphson Iteration [3], which could have dangerous consequences (particularly when used in cryptographic applications) if remained undetected. 


\begin{tabular}{c|r|r|r|r|r|c|c|c} 
& Proc. & \multicolumn{1}{|c|}{ Lem. } & Rules & User & System & $\%$ & Steps & mm:ss \\
\hline $\mathfrak{I}_{B}(n, m)=n_{m}^{-1}$ & $8(7)$ & $49(3)$ & $241(39)$ & $36(3)$ & $205(36)$ & $85,1(92,3)$ & 3171 & $0: 19$ \\
$\mathfrak{I}_{N}(n, m)=n_{m}^{-1}$ & $10(9)$ & $76(3)$ & $368(59)$ & $59(3)$ & $309(56)$ & $84,0(94,9)$ & 6692 & $1: 32$ \\
Theorems 1 \&2 2 & $20(12)$ & $116(3)$ & $547(78)$ & $96(6)$ & $451(72)$ & $82,4(92,3)$ & 9739 & $2: 19$
\end{tabular}

Fig. 7. Proof statistics

Figure 7 displays the effort for obtaining the proofs (including all procedures and lemmas which had been imported from our arithmetic proof library). Column Proc. counts the number of user defined procedures (the recursively defined ones given in parentheses), Lem. is the number of user defined lemmas (the number of synthesized lemmas given in parentheses), and Rules counts the total number of $H P L$-proof rule applications, separated into user invoked (User) and system initiated (System) ones (with the number of uses of Induction given in parentheses). Column \% gives the automation degree, i.e. the ratio between System and Rules, Steps lists the number of first-order proof steps performed by the Symbolic Evaluator and Time displays the runtime of the Symbolic Evaluator. ${ }^{8}$

The first two rows show the effort for proving Lemmas 9 and 20 as illustrated in Sect. 3. As it can be observed from the numbers, verifying the computation of multiplicative inverses by Newton-Raphson Iteration is much more challenging for the system and for the user than the method based on Bézout's Lemma. Row Theorems 1 and 2 below displays the effort for proving Theorems 1 and 2 as illustrated in Sect. 4 (with the effort for the proofs of Lemmas 9 and 20 included).

The numbers in Fig. 7 almost coincide with the statistics obtained for other case studies in Number Theory performed with the system (see e.g. [14] and also [7] for more examples), viz. an automation degree of $\sim 85 \%$ and a success rate of $\sim 95 \%$ for the induction heuristic. All termination proofs (hence all required induction axioms in turn) had been obtained without user support, where 6 of the 12 recursively defined procedures, viz. mod, /, gcd, $\log _{2}$, euclid and $\mathfrak{I}_{N^{\prime}}$, do not terminate by structural recursion. ${ }^{9}$ While an automation degree up to $100 \%$ can be achieved in mathematically simple domains, e.g. when sorting lists $[7,9]$, values of $85 \%$ and below are not that satisfying when concerned with automated reasoning. The cause is that quite often elaborate ideas for developing a proof are needed in Number Theory which are beyond the ability of the system's heuristics guiding the proof search. ${ }^{10}$ We also are not aware of other reasoning systems offering more machine support for obtaining proofs in this difficult domain.

8 Time refers to running VeriFun 3.5 under Windows 7 Enterprise with an INTEL Core i7-2640M 2.80 GHz CPU using Java 1.8.0_45.

${ }^{9}$ Procedure $2^{?}(\ldots)$ is not user defined, but synthesized as the domain procedure [12] of the incompletely defined procedure $\log _{2}$.

${ }^{10}$ Examples are the use of the quotient-remainder theorem for proving (i) in Sect. 3.1 and (iii) in Sect. 3.2 which are the essential proof steps there although more complex proof obligations result. 
From the user's perspective, this case study necessitated more work than expected, and it was a novel experience for us to spend some effort for verifying a very small and non-recursively defined procedure. The reason is that correctness of procedure redc depends on some non-obvious and tricky number theoretic principles which made it difficult to spot the required lemmas. In fact, almost all effort was spend for the invention of the auxiliary lemmas in Sect. 4 and of Lemma 12 in Sect.3.1. Once the "right" lemma for verifying a given proof obligation eventually was found, its proof turned out to be a routine task. The proof of Lemma 17 is an exception as it required some thoughts to create it and some effort as well to lead the system (thus spoiling the proof statistics). Proof development was significantly supported by the system's Disprover [1] which (besides detecting the fault in Algorithm 2') often helped not to waste time with trying to prove a false conjecture, where the computed counterexamples provided useful hints how to debug a lemma draft.

\section{References}

1. Aderhold, M., Walther, C., Szallies, D., Schlosser, A.: A fast disprover for $\sqrt{e r i F u n . ~}$ In: Ahrendt, W., Baumgartner, P., de Nivelle, H. (eds.) Proc. Workshop on NonTheorems, Non-Validity, Non-Provability, DISPROVING 2006, Seattle, WA, pp. 59-69 (2006). http://verifun.de/documents

2. Brent, R., Zimmermann, P.: Modern Computer Arithmetic. Cambridge University Press, New York (2010)

3. Dumas, J.: On Newton-Raphson iteration for multiplicative inverses modulo prime powers. IEEE Trans. Comput. 63(8), 2106-2109 (2014). https://doi.org/10.1109/ TC.2013.94

4. Hankerson, D., Menezes, A.J., Vanstone, S.: Guide to Elliptic Curve Cryptography. Springer, Secaucus (2003). https://doi.org/10.1007/b97644

5. Menezes, A.J., Oorschot, P.C.V., Vanstone, S.A., Rivest, R.L.: Handbook of Applied Cryptography. CRC Press Inc., Boca Raton (2001)

6. Montgomery, P.L.: Modular multiplication without trial division. Math. Comput. 44(170), 519-521 (1985). https://doi.org/10.1090/S0025-5718-1985-0777282-X

7. VeriFun. http://www.verifun.de

8. Walther, C.: On proving the termination of algorithms by machine. Artif. Intell. 71(1), 101-157 (1994). https://doi.org/10.1016/0004-3702(94)90063-9

9. Walther, C.: A largely automated verification of GHC's natural mergesort. Technical report VFR 17/01, FB Informatik, Techn. Universität Darmstadt (2017)

10. Walther, C., Schweitzer, S.: Verification in the classroom. J. Autom. Reason. 32(1), 35-73 (2004). https://doi.org/10.1023/B:JARS.0000021872.64036.41

11. Walther, C., Schweitzer, S.: Automated termination analysis for incompletely defined programs. In: Baader, F., Voronkov, A. (eds.) LPAR-11. LNCS, vol. 3452, pp. 332-346. Springer, Heidelberg (2005). https://doi.org/10.1007/978-3540-32275-7_22

12. Walther, C., Schweitzer, S.: Reasoning about incompletely defined programs. In: Sutcliffe, G., Voronkov, A. (eds.) LPAR-12. LNCS, vol. 3835, pp. 427-442. Springer, Heidelberg (2005). https://doi.org/10.1007/11591191_30 
13. Walther, C., Schweitzer, S.: A pragmatic approach to equality reasoning. Technical report VFR 06/02, FB Informatik, Technische Universität Darmstadt (2006). http://verifun.de/documents

14. Walther, C., Wasser, N.: Fermat, Euler, Wilson - Three case studies in number theory. J. Autom. Reason. 59(2), 267-286 (2017). https://doi.org/10.1007/s10817$016-9387-z$

Open Access This chapter is licensed under the terms of the Creative Commons Attribution 4.0 International License (http://creativecommons.org/licenses/by/4.0/), which permits use, sharing, adaptation, distribution and reproduction in any medium or format, as long as you give appropriate credit to the original author(s) and the source, provide a link to the Creative Commons license and indicate if changes were made.

The images or other third party material in this chapter are included in the chapter's Creative Commons license, unless indicated otherwise in a credit line to the material. If material is not included in the chapter's Creative Commons license and your intended use is not permitted by statutory regulation or exceeds the permitted use, you will need to obtain permission directly from the copyright holder.

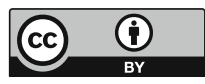

